\title{
Maternal Diet and Neonatal Body Composition
}

\author{
L. Mullaney ${ }^{1}$, A. O’Higgins ${ }^{2}$, A. Doolan ${ }^{3}$, M. Sheridan-Pereira ${ }^{3}$, D. McCartney ${ }^{1}$ \\ and M. J. Turner ${ }^{2}$ \\ ${ }^{1}$ School of Biological Sciences, Dublin Institute of Technology, Dublin 8, Republic of Ireland, ${ }^{2}$ UCD Centre for Human \\ Reproduction, CWIUH, Dublin 8, Republic of Ireland and ${ }^{3}$ Department of Pediatrics, Trinity College, Dublin 2, \\ Republic of Ireland
}

There is now considerable evidence that maternal nutritional intake may programme fetal development. For example, low iron status in pregnancy has been linked to low birth weight and impaired cognitive development ${ }^{(1,2,3)}$. In addition to this, children born to obese women have been shown to be at increased risk of obesity and diabetes in adolescence and later adult life ${ }^{(4,5)}$. However, most studies use birth weight as a surrogate marker to evaluate intra-uterine nutritional status and prospective neonatal health outcomes. Studies investigating the association between maternal nutritional intake and neonatal body composition are lacking.

This prospective observational study examined the relationship between maternal nutritional intake in the first trimester and neonatal body composition.

Women were recruited in February 2013 after sonographic confirmation of a singleton pregnancy in the first trimester. Clinical and socio-demographic details were recorded and maternal body mass index (BMI) measured. Dietary information was collected at the same visit using a Willett Food Frequency Questionnaire (WFFQ) previously validated in pregnancy ${ }^{(6)}$. Neonatal body composition was measured within three days of delivery using air displacement plethysmography.

Of the 75 mother-infant pairs, the mean maternal BMI was $25 \cdot 1 \mathrm{~kg} / \mathrm{m}^{2}( \pm 5 \cdot 7)$ and $14 \cdot 8 \%$ were obese. The mean birth weight was $3.5 \mathrm{~kg}( \pm 0.5)$ and mean percentage body fat was $10.8 \%( \pm 4 \cdot 5)$. Mean percentage energy intakes from protein, fat, and carbohydrate were $17 \cdot 2 \%, 35 \cdot 4 \%$, and $49 \cdot 8 \%$ respectively. There was no association between birth weight and maternal energy intakes from protein, fat, or carbohydrate. However the mean infant body fat percentage for mothers in the lowest fat consumption quartile was $8.7 \%$ compared to $12.5 \%$ in the highest quartile $(p=0.02)$. Mean infant body fat percentage for mothers in the lowest carbohydrate consumption quartile was $13 \cdot 1 \%$ compared to $9 \cdot 1 \%$ in the highest quartile $(p=0.003)$. Neonatal adiposity was not associated with maternal protein intake.

These findings suggest that interventions that decrease maternal fat intake in early pregnancy may decrease neonatal adiposity and thus, potentially, the lifelong risk of metabolic dysfunction.

1. Haider BA, Olofin I, Wang M, Spiegelman D, Ezzati M, Fawzi WW. (2013) BMJ 346, f3443.

2. Tran TD, Biggs BA, Tran T, Simpson JA, Hanieh S, Dwyer T, Fisher J. (2013) PLoS One 8, e74876.

3. Radlowski EC \& Johnson RW. (2013) Front Hum Neurosci 7, 585.

4. Baker JL, Michaelsen KF, Rasmussen KM, Sorensen TI. (2004) Am J ClinNutr 80, 1579-1588

5. Satpathy HK, Fleming A, Frey D, Barsoom M, Satpathy C, Khandalavala J (2008) Postgrad Med 120, 01-09.

6. McGowan CA, Curran S, McAuliffe FM (2013) J Hum Nutr Diet [Epub ahead of print]. 